\title{
Comparison of corneal optical quality after SMILE, wavefront-optimized LASIK and topography-guided LASIK for myopia and myopic astigmatism
}

Yu Zhang

Peking University Third Hospital

Xiaoxiao Sun

Peking University Third Hospital

Yueguo Chen ( 1494867399@qq.com )

Peking University Third Hospital https://orcid.org/0000-0003-2720-7135

\section{Research Article}

Keywords: SMILE, FS-LASIK, optical quality, topography-guided

Posted Date: April 30th, 2021

DOI: https://doi.org/10.21203/rs.3.rs-308202/v1

License: (c) (1) This work is licensed under a Creative Commons Attribution 4.0 International License.

Read Full License

Version of Record: A version of this preprint was published at Frontiers in Medicine on April 5th, 2022. See the published version at https://doi.org/10.3389/fmed.2022.870330. 


\section{Abstract}

Purpose: To compare visual outcomes and corneal optical quality after small incision lenticule extraction (SMILE) , wavefront-optimized (WFO) FS-LASIK and topography-guided customized ablation treatment (TCAT) FS-LASIK for myopia.

Methods: This prospective study included 283 eyes of 283 myopic patients who underwent SMILE or FSLASIK according to the patient's wishes. FS-LASIK patients were randomly assigned to use WFO or TCAT ablation. There were 102 eyes, 100 eyes and 81eyes in the SMILE group, WFO group and TCAT group, respectively. The combined corneal topographer and tomographer system (Sirius) was used to measure corneal aberrations and optical quality. Visual outcomes and corneal aberrations were compared among the three groups.

Results: At postoperative 1 and 6 months, there were no significant differences in uncorrected distance visual acuity (UDVA) and corrected distance visual acuity (CDVA) among the three groups $(P>.05)$. Postoperative manifest refractive spherical equivalent was similar among the groups $(P>.05)$. There was statistically significant difference in cylinder at 1 month among the three groups, with the highest mean value in TCAT group $(P<.05)$. The corneal optical path difference (OPD), root mean square of corneal astigmatism and strehl ratio (SR) were the most superior in the TCAT group at postoperative 1 and 6 months $(P<.05)$.

Conclusion: SMILE, WFO FS-LASIK and TCAT FS-LASIK provided similar visual results. TCAT FS-LASIK could induce fewer corneal OPD and astigmatism, and higher SR than the others. However, a better algorithm for TCAT FS-LASIK is needed to decrease postoperative residual astigmatism.

\section{Introduction}

SMILE and FS-LASIK, which have been well proved to be effective and safe, are two main stream surgical procedures of myopia and myopic astigmatism correction ${ }^{1-2}$. It is controversial about which of the two surgical methods is better for vision and visual quality. And no matter which type of surgery is used, some patients still have problems of visual quality after operation. Studies have shown that this may be related to the size of the optical zone, the state of refraction, the laser ablation profile, the centering of ablation, and the postoperative morphology of corneal aspheric surface and regularity ${ }^{3-5}$. In order to improve the postoperative visual quality, various improved laser ablation algorithms such as wavefrontoptimized (WFO), topography-guided customized ablation treatment (TCAT) have been developed in excimer laser ablation profile of FS-LASIK.

WFO ablation attempts to reduce the induction of spherical aberration by adding peripheral pulses, blending it with the central ablation profile and maintaining the prolate shape of the cornea ${ }^{6}$. TCAT ablation attempts to maintain the aspheric shape of the cornea and neutralize corneal irregularities ${ }^{7}$. 
The SMILE has only one symmetric spherical ablation profile without automatic kappa angle and cyclotorsion compensation. In spite of the relative merits of SMILE contrast to FS-LASIK on less dry eye and potential flap complications, a thorough comparison of these two procedures is helpful for clinicians as they navigate which treatment to choose in patient care. To our knowledge, this is the first study comparing SMILE, WFO LASIK and TCAT LASIK simultaneously regarding vision, refraction, corneal aberrations and optical quality.

\section{Patients And Methods}

\section{Patients}

Two hundred and eighty-three eyes of 283 patients who underwent bilateral myopia and myopic astigmatism correction with FS-LASIK or SMILE at Peking University Third Hospital from 2017 to 2019 were included in this prospective study.

Inclusion criteria were age $\geq 18$ years, myopic sphere up to $8.00 \mathrm{D}$, cylinder up to $1.50 \mathrm{D}$, with a documented refractive stability for a minimum period of 1 year and discontinuation of soft contact lenses for at least 2 weeks. Exclusion criteria included a residual stromal bed less than $280 \mu \mathrm{m}$, topographic evidence of corneal ectasia, noted complications during and after surgical procedure, previous ocular surgery, history of herpetic eye disease, collagen vascular disease, pregnancy and lactation.

In accordance with each patient's wish, SMILE was performed in 102 patients (SMILE group) and FSLASIK was performed in 181 patients. Among FS-LASIK, 100 patients were randomly assigned to use WFO ablation profile (WFO group) and the other 81 patients were assigned to use TCAT profile (TCAT group). One eye of each patient was randomly chosen for analysis.

The study received approval from the Ethics Committee of our institute and was conducted in accordance with the tenets of the Declaration of Helsinki. A written informed consent was obtained from each patient prior to the surgical procedure.

\section{Preoperative examinations}

Preoperative evaluation included uncorrected distance visual acuity (UDVA) and corrected distance visual acuity (CDVA), manifest refraction, slit lamp bio-microscopy, dilated fundus evaluation, corneal thickness

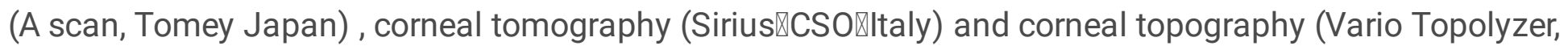
WaveLight, Alcon Laboratories, Inc., Fort Worth, TX). The total corneal aberrations and optic quality in a 6$\mathrm{mm}$ zone were obtained from corneal tomography. Parameters of corneal aberrations and optical quality included optical path difference (OPD), root mean square of higher order aberration (RMSh), RMS of corneal astigmatism, RMS of spherical aberration (SA), RMS of coma and strehl ratio (SR). Corneal topography data of kappa angle, static cyclotorsion compensation for excimer laser ablation and anterior 
corneal map for topography guided procedure were obtained from the Vario Topolyzer placido-based topography.

\section{Surgical procedures}

All surgeries were performed under topical anesthesia by an experienced refractive surgeon (YG Chen). For FS-LASIK, all flaps were created by the WaveLight FS200 femtosecond laser. The flap/canal/hinge parameters were as followed: flap thickness, $110 \mu \mathrm{m}$; flap diameter, $8.5 \mathrm{~mm}$ to $9.0 \mathrm{~mm}$; side-cut angle, $90^{\circ}$; hinge angle, $50^{\circ}$; canal width, $1.5 \mathrm{~mm}$. Following blunt dissection and flap lift, the stromal bed was ablated with excimer laser (EX500 WaveLight) using an optic zone of $6.5 \mathrm{~mm}$ with a $1.25 \mathrm{~mm}$ transition zone. The refraction data (sphere, cylinder and axis) used for the eyes in the WFO group was the subjective manifest refraction. The refraction data used for the eyes in the TCAT group was partially followed topography-modified refraction (TMR) scheme introduced by AJ Kanellopoulos ${ }^{8}$.

SMILE was performed with the VisuMax femtosecond laser system (Zeiss, Germany, Nomogram version 3.0). The energy setting was $140 \mathrm{~nJ}$, and the laser spot spacing was $4.5 \mathrm{~mm}$ for the lenticule and cap interface, $2.0 \mathrm{~mm}$ for the lenticule side cut and cap side cut. The spherical refraction data was $10 \%$ more on the basis of manifest refraction. The lenticule base thickness was $10 \sim 15 \mathrm{~mm}$. The cap thickness was $120 \mathrm{~mm}$, and the cap diameter was $7.6 \mathrm{~mm}$ with $2 \mathrm{~mm}$ small incision width. The lenticule optical zone (OZ) was $6.5 \mathrm{~mm}$ with $0.1 \mathrm{~mm}$ transition zone for astigmatism.

\section{Postoperative care and follow-up}

Postoperatively, all the eyes received treatment with $0.1 \%$ fluorometholone (FML, Allergan, Inc.) in tapering dose for 4 weeks, $0.5 \%$ levofloxacin (Cravit, Santen, Inc.) four times a day for 2 weeks and lubricating drops four times a day for 4 weeks. Follow-up visits included postoperative day 1 and 7 , month 1, 3 and 6 . The follow-up examinations involved measurements of UDVA, slit-lamp examination, manifest refraction, CDVA and corneal tomography (Sirius, CSO, Italy).

\section{Statistical analysis}

Data were analyzed using SPSS software (version 21.0; SPSS, Inc., Chicago, IL). The KolmogorovSmirnov test was used for confirming normality of data. All the data were represented as mean \pm standard deviation. The normally distributed data were compared among the three groups using One-way ANOVA. The non-normally distributed data were compared among the three groups using Kruskal-Wallis $\mathrm{H}$ test. Comparisons of the distribution of visual acuity and refraction among the three groups were analyzed by Pearson chi-square test. A $P$ value of less than .05 was considered statistically significant.

\section{Results}

This study included 283 eyes of 283 patients. There were 102 eyes, 100 eyes and 81 eyes in the SMILE group, WFO group and TCAT group, respectively. There was no statistically significant difference among 
the three groups regarding age, sex, preoperative refraction, corneal aberrations, and Strehl ratio $(P>.05)$ (Table 1).

\section{UDVA outcome}

At postoperative 1 month, UDVA of $20 / 20$ or better was measured in $95.1 \%$ of eyes in the SMILE group, $97.0 \%$ of eyes in the WFO group and $93.8 \%$ of eyes in the TCAT group $(P=.393)$ (Figure 1A).

At postoperative 6 months, UDVA of $20 / 20$ or better was measured in $96.1 \%$ of eyes in the SMILE group, $97.0 \%$ of eyes in the WFO group and $95.1 \%$ of eyes in the TCAT group $(P=.658)$ (Figure 1B).

\section{CDVA outcome}

At postoperative 1 month, $10.8 \%$ of the eyes in the SMILE group, $17.0 \%$ in the WFO group and $9.9 \%$ in the TCAT group gained one line of CDVA. A lose of one line was noted in $5.9 \%, 7.0 \%$ and $9.9 \%$ of the eyes in the SMILE, WFO and TCAT groups, respectively. No change in line was noted in $78.4 \%, 61.0 \%$ and $71.6 \%$ of the eyes in the SMILE, WFO and TCAT groups, respectively $(P=.083)$ (Figure 2A).

At postoperative 6 months, $9.8 \%$ of the eyes in the SMILE group, $16.0 \%$ in the WFO group and $13.6 \%$ in the TCAT group gained one line of CDVA. A lose of one line was noted in $6.9 \%, 5.0 \%$ and $3.7 \%$ of the eyes in the SMILE, WFO and TCAT groups, respectively. No change in line was noted in $71.6 \%, 60.0 \%$ and $65.4 \%$ of the eyes in the SMILE, WFO and TCAT groups, respectively $(P=.501)$ (Figure 2B).

\section{Refractive outcome}

Postoperative MRSE and cylinder at 1 and 6 months are shown in table 2.There was statistically significant difference in cylinder at 1 month among the three groups. $(P<.05)$ Meanwhile, the mean cylinder at 1 month in the WFO group $(-0.17 \pm 0.23 \mathrm{D})$ was significant less than that in the TCAT group $(-0.27 \pm 0.28 \mathrm{D})(P<.05)$.

At postoperative 1 month, the residual MRSE within $\pm 0.13 \mathrm{D}$ was achieved by $55.9 \%$ of eyes in the SMILE group, $55.0 \%$ of eyes in the WFO group and $48.1 \%$ of eyes in the TCAT group $(P=.543)$ (Figure 3A). At postoperative 6 months, the residual MRSE within $\pm 0.13 \mathrm{D}$ was achieved by $54.9 \%$ of eyes in the SMILE group, $64.0 \%$ of eyes in the WFO group and $54.3 \%$ of eyes in the TCAT group $(P=.127)$ (Figure 3B).

Postoperative refractive astigmatism is noted in Figure 4. At 1 month, $76.5 \%$ of eyes in the SMILE group, $76.0 \%$ of eyes in the WFO group and $65.4 \%$ of eyes in the TCAT group were $0.25 \mathrm{D}$ or less. There was no statistically significant difference among the three groups $(P=.095)$. At 6 months, $76.5 \%$ of eyes in the SMILE group, $82.0 \%$ of eyes in the WFO group and $69.2 \%$ of eyes in the TCAT group were 0.25 D or less $(P$ $=.055)$.

\section{Corneal aberrations and optical quality}


At both 1 month and 6 months, OPD and RMS of corneal astigmatism in the TCAT group were significantly lower than the other two groups $(P<.05)$. However, there were no statistically significant differences in RMSh, RMS of coma or SA among the three groups $(P>.05)$. At postoperative 1 month, SR in the TCAT group was significantly higher than that in the other two groups $(P<.05)$. At 6 months, SR was significantly different among the three groups $(P<.05)$, the highest in the TCAT group, the lowest in the SMILE group, and the middle in the WFO group $(P<.05)$. 8 Table 3 and $4 \rrbracket$

\section{Discussion}

The current study found no significant differences in postoperative UDVA or CDVA among SMILE, WFO and TCAT groups, showing that the three procedures were comparably effective and safe. The majority of previous studies demonstrated the similar results ${ }^{9-14}$. A randomized, paired-eye study found that SMILE achieved similar results as WFO LASIK in terms of efficacy index $(0.97 \pm 0.20 \mathrm{vs.} 0.99 \pm 0.20 ; P=0.56)$, UDVA of $20 / 40$ or better ( $100 \%$ vs. $100 \% ; P=1.0)$, and UDVA of $20 / 20$ or better $(84 \% \text { vs. } 87 \% ; P=0.63)^{9}$. However, a prospective, randomized contralateral eye study found that $86.4 \%$ of the topography-guided LASIK group and $68.2 \%$ of the SMILE group had UDVA of $20 / 20(P<.002)$ and $59.1 \%$ and $31.8 \%$, respectively, had UDVA of 20/16 $(P<.002)$ at 3 months $^{15}$. The inferior visual performance of SMILE was due to higher residual spherical and cylinder refraction. The present study showed a much better visual results in SMILE group than this study.

The present study found that postoperative MRSE at 1 and 6 months was similar among the three groups, but the 1-month postoperative cylinder was significantly different among the three groups, with the highest mean value in the TCAT group. Most previous studies showed comparable refractive results between SMILE and WFO FS-LASIK or SMILE and TCAT FS-LASIK ${ }^{9-14}$. The high residual cylinder in the TCAT group of the present study was similar to our previous study ${ }^{16}$, which might be caused by the compensation method for TCAT design. In the present study, partial TMR was applied to the TCAT design of cylinder and axis. However, Kanellopoulos AJ ${ }^{15}$ compared SMILE and Topo-LASIK with TMR method and found that Topo LASIK was superior in postoperative MRSE and cylinder to SMILE. The higher residual refraction in SMILE might be caused by inappropriate nomogram of parameter design, high preoperative cylinder, lack of eye tracking and cyclotorsion compensation. Better design methods for TCAT are needed to obtain more accurate refractive results. The Phorcides Analytic Engine for topography-guided surgery planning was preliminarily proved to have good refractive results, with $83 \%$ of eyes showing a refractive cylinder of less than $0.25 \mathrm{D}$ postoperatively ${ }^{17}$, which needs further more extensive verification in the future.

Most of aberrations come from cornea. Good corneal optical quality is the premise of the visual quality. The present study also found that corneal OPD, RMS of corneal astigmatism and SR were the most superior in the TCAT group, showing that corneal optical quality was the best after TCAT LASIK. To the best of our knowledge, no previous study compared the corneal aberrations and optical qualities among the three procedures. The lowest OPD in the TCAT group mainly derived from the lowest RMS of corneal 
astigmatism, but not from the higher-order aberrations. Several studies showed fewer corneal coma after wavefront-guided LASIK compared to SMILE, which might be due to automatic eye tracking with active centration control and cyclotorsion compensation in LASIK ${ }^{18-19}$. In the present study, however, RMS of coma was comparably low in the TCAT group, but the difference wasn't statistically significant. Some studies also found lower corneal SA after SMILE compared to FS-LASIK ${ }^{11,13,20}$. As we known, SMILE procedure could achieve a larger optical zone than do FS-LASIK procedures because of less biomechanical alterations in the peripheral area of the cornea after SMILE ${ }^{20}$. Postoperative spherical aberration was associated with optical and ablation zones ${ }^{21}$. Yet, in the present study, postoperative SA in the SMILE group was comparably low, but compared with the other two groups, there was no significant difference. Similar with the present study, El-Mayah et al. also found a similar comparative trend of postoperative coma and SA between SMILE and Fs-LASIK $(P>0.05)^{22}$. In the future, larger sample, randomized controlled studies are needed to further compare corneal aberrations and optical quality among the three surgical procedures.

The limitation of our study is that we only compared visual acuity, refraction, corneal aberrations and optical qualities among the three groups, and other parameters related with visual quality, such as contrast-sensitivity function and Objective Scatter Index weren't studied.

In conclusion, SMILE, wavefront optimized FS-LASIK and topography guided FS-LASIK provided similar visual results. Topography guided FS-LASIK could induce fewer corneal optical path difference and astigmatism, and higher strehl ratio than the others. However, topography guided FS-LASIK could induce more manifest residual cylinder and a more accurate algorithm for compensating the irregular ablation, corneal posterior surface and internal eye astigmatisms is needed to further improve postoperative visual acuity and refractive outcomes.

\section{Declarations}

\section{Funding Info}

Capital's Funds for Health Improvement and Research (2018-2-4092)

\section{Competing interests}

The authors declare that they have no competing interests

\section{Availability of data and materials}

The datasets used and/or analyzed during the current study are available from the corresponding author on reasonable request.

\section{Code availability}

Not applicable 


\section{Authors' contributions}

$\mathrm{YZ}$ analyzed and interpreted the patient data, and wrote the manuscript. XS collected and analyzed the data. YC designed the study and revised the manuscript. All authors read and approved the final manuscript.

\section{Ethics approval}

This study was carried out in accordance with the tenets of the Declaration of Helsinki. It was approved by the Ethics Committee of Peking University Third Hospital.

\section{consent to participate}

An informed consent was obtained from each subject.

\section{Consent for publication}

Not applicable

\section{References}

1. Sekundo W, Kunert K, Russmann C, et al. First efficacy and safety study of femtosecond lenticule extraction for the correction of myopia: six-month results. J Cataract Refract Surg. 2008;34(9):15131520.

2. Schallhorn JM, Seifert S, Schallhorn SC. SMILE, topography-guided LASIK: review of clinical outcomes in premarket approval FDA studies. J Refract Surg. 2019; 35(11):690-698.

3. Mok KH, Lee VW. Effect of optical zone ablation diameter on LASIK-induced higher order optical aberrations. J Refract Surg. 2005; 21(2):141-143.

4. Oshika T, Tokunaga T, Samejima T, et al. Influence of pupil diameter on the relation between ocular higher-order aberration and contrast sensitivity after laser in situ keratomileusis. Invest Ophthalmol Vis Sci. 2006; 47(4):1334-1338.

5. Villa C, Gutiérrez R, Jiménez JR, González-Méijome JM. Night vision disturbances after successful LASIK surgery. Br J Ophthalmol. 2007;91(8):1031-1037.

6. Mrochen M, Donitzky C, Wüllner C, Löffler J. Wavefront-optimized ablation profiles: theoretical background. J Cataract Refract Surg. 2004;30(4):775-785.

7. Schallhorn SC, Farjo AA, Huang D, et al; American Academy of Ophthalmology. Wavefront-guided LASIK for the correction of primary myopia and astigmatism a report by the American Academy of Ophthalmology. Ophthalmology. 2008;115(7):1249-1261.

8. Kanellopoulos AJ. Topography-modified refraction(TMR): adjustment of treated cylinder amount and axis to the topography versus standard clinical refraction in myopic topography-guided LASIK. Clinical Ophthalmology. 2016;10:2213-2221. 
9. Ang M, Farook M, Htoon HM, Mehta JS. Randomized clinical trial comparing Femtosecond LASIK and Small-Incision Lenticule Extraction. Ophthalmology. 2020;127(6):724-730.

10. Schallhorn JM, Seifert S, Schallhorn SC. SMILE, Topography-Guided LASIK, and Wavefront-Guided LASIK: Review of Clinical Outcomes in Premarket Approval FDA Studies. J Refract Surg. 2019;35(11):690-698.

11. Liu M, Chen Y, Wang D, et al. Clinical outcomes after SMILE and femtosecond laser-assisted LASIK for myopia and myopic astigmatism: a prospective randomized comparative study. Cornea. 2016;35(2):210-216.

12. Li M, Li M, Chen Y, Miao H, Yang D, Ni K, Zhou X. Five-year results of small incision lenticule extraction (SMILE) and femtosecond laser LASIK (FS-LASIK) for myopia. Acta Ophthalmol. 2019;97(3):e373-e380.

13. Han T, Xu Y, Han X, Zeng L, Shang J, Chen X, Zhou X. Three-year outcomes of small incision lenticule extraction (SMILE) and femtosecond laser-assisted laser in situ keratomileusis (FS-LASIK) for myopia and myopic astigmatism.Br J Ophthalmol. 2019;103(4):565-568.

14. Moshirfar M, Somani AN, Motlagh MN, et al. Comparison of FDA-Reported Visual and Refractive Outcomes of the Toric ICL Lens, SMILE, and Topography-Guided LASIK for the Correction of Myopia and Myopic Astigmatism. J Refract Surg. 2019;35(11):699-706.

15. Kanellopoulos AJ. Topography-Guided LASIK Versus Small Incision Lenticule Extraction (SMILE) for Myopia and Myopic Astigmatism: A Randomized, Prospective, Contralateral Eye Study. J Refract Surg. 2017;33(5):306-312.

16. Zhang Y, Chen YG. A Randomized Comparative Study of Topography-Guided Versus Wavefront Optimized FS-LASIK for Correcting Myopia and Myopic Astigmatism. J Refract Surg. 2019; 35(9): 575-582.

17. Lobanoff M, Stonecipher K, Tooma T, Wexler S, Potvin R. Clinical outcomes after topography-guided LASIK: comparing results based on a new topography analysis algorithm with those based on manifest refraction. J Cataract Refract Surg. 2020;46(6):814-819.

18. Chen X, Wang Y, Zhang J, et al. Comparison of ocular higher-order aberrations after SMILE and Wavefront guided Femtosecond LASIK for myopia. BMC Ophthalmology 2017; 17:42.

19. XiaLK, MaJ, Liu HN, Shi C, Huang Q. Three-year results of small incision lenticule extraction and wavefront-guided femtosecond laser-assisted laser in situ keratomileusis for correction of high myopia and myopic astigmatism. Int J Ophthalmol. 2018;11(3):470-477.

20. Damgaard IB, Ang M, Mahmoud AM, Farook M, Roberts CJ, Mehta JS. Functional Optical Zone and Centration Following SMILE and LASIK: A Prospective, Randomized, Contralateral Eye Study. J Refract Surg. 2019;35(4):230-237.

21. Vega-Estrada A, Alio JL, Arba MS, et al. Corneal higher order aberrations after LASIK for high myopia with a fast repetition rate excimer laser, optimized ablation profile, and femtosecond laser-assisted flap. J Refract Surg. 2012;28:689-696. 
22. El-Mayah E, Anis M, Salem M, Pinero D, Hosny M. Comparison Between Q-Adjusted LASIK and SmallIncision Lenticule Extraction for Correction of Myopia and Myopic Astigmatism. Eye \& Contact Lens. 2018 ;44 Suppl 2:S426-S432.

\section{Tables}

Table 1 Baseline clinical data and demographics in the three groups

\begin{tabular}{|c|c|c|c|c|c|}
\hline & SMILE $(n=102)$ & WFO $(n=100)$ & $\operatorname{TCAT}(\mathrm{n}=81)$ & $\mathrm{F} / \chi^{2}$ & $P$ \\
\hline Male/Female & $22 / 80$ & $24 / 76$ & $15 / 66$ & & \\
\hline Right/Left & $51 / 51$ & $54 / 46$ & $35 / 46$ & & \\
\hline Sphere(D) & $-4.96 \pm 1.72$ & $-4.95 \pm 1.57$ & $-5.22 \pm 1.49$ & 0.758 & 0.470 \\
\hline Cylinder(D) & $-0.63 \pm 0.31$ & $-0.63 \pm 0.38$ & $-0.65 \pm 0.37$ & 0.110 & 0.896 \\
\hline $\mathrm{OPD}(\mu \mathrm{m})$ & $0.88 \pm 0.35$ & $0.93 \pm 0.41$ & $0.98 \pm 0.39$ & 1.550 & 0.214 \\
\hline $\mathrm{RMSh}(\mu \mathrm{m})$ & $0.41 \pm 0.13$ & $0.42 \pm 0.13$ & $0.44 \pm 0.15$ & 0.763 & 0.467 \\
\hline RMS-AST( $\mu \mathrm{m})$ & $0.75 \pm 0.40$ & $0.80 \pm 0.45$ & $0.86 \pm 0.42$ & 1.527 & 0.219 \\
\hline RMS-coma $(\mu \mathrm{m})$ & $0.22 \pm 0.13$ & $0.22 \pm 0.13$ & $0.25 \pm 0.15$ & 1.417 & 0.244 \\
\hline RMS-SA( $\mu \mathrm{m})$ & $0.22 \pm 0.07$ & $0.22 \pm 0.07$ & $0.23 \pm 0.08$ & 0.683 & 0.506 \\
\hline Strehl Ratio & $0.16 \pm 0.08$ & $0.15 \pm 0.06$ & $0.14 \pm 0.05$ & 0.977 & 0.378 \\
\hline
\end{tabular}

SMILE: small incision lenticule extraction; WFO: Wavefront-optimized laser in situ keratomileusis; TCAT: Topography-guided custom laser in situ keratomileusis; OPD: optical path difference; RMSh: root mean square of higher order aberrations; AST: corneal astigmatism; SA: spherical aberration

Table 2 Comparison of postoperative manifest refraction among the three groups.

\begin{tabular}{lccccc}
\hline & SMILE(n=102) & WFO(n=100) & TCAT $(\mathrm{n}=81)$ & $\chi^{2}$ & $P$ \\
\hline MRSE 1M(D) & $-0.02 \pm 0.30$ & $0.03 \pm 0.31$ & $0.07 \pm 0.39$ & 5.293 & 0.071 \\
MRSE 6M(D) & $-0.06 \pm 0.27$ & $-0.06 \pm 0.23$ & $0.01 \pm 0.28$ & 3.445 & 0.179 \\
Cylinder 1M(D) & $-0.21 \pm 0.24$ & $-0.17 \pm 0.23^{*}$ & $-0.27 \pm 0.28^{*}$ & 6.655 & 0.036 \\
Cylinder 6M(D) & $-0.19 \pm 0.23$ & $-0.18 \pm 0.24$ & $-0.22 \pm 0.25$ & 1.959 & 0.375 \\
\hline
\end{tabular}

*: The value was significantly different from that in the other two groups.

SMILE: small incision lenticule extraction; WFO: Wavefront-optimized laser in situ keratomileusis; TCAT: Topography-guided custom laser in situ keratomileusis; MRSE: manifest refractive spherical equivalent 
Table 3 Comparison of corneal aberrations and visual quality among the three groups at 1 month postoperatively

\begin{tabular}{|c|c|c|c|c|c|}
\hline & SMILE(n=102) & $\mathrm{WFO}(\mathrm{n}=100)$ & $\operatorname{TCAT}(\mathrm{n}=81)$ & F & $P$ \\
\hline $\mathrm{OPD}(\mu \mathrm{m})$ & $0.92 \pm 0.40$ & $0.90 \pm 0.31$ & $0.77 \pm 0.28 *$ & 5.028 & 0.007 \\
\hline $\mathrm{RMSh}(\mu \mathrm{m})$ & $0.67 \pm 0.26$ & $0.69 \pm 0.25$ & $0.67 \pm 0.27$ & 0.305 & 0.738 \\
\hline RMS-AST( $\mu \mathrm{m})$ & $0.57 \pm 0.41$ & $0.52 \pm 0.30$ & $0.33 \pm 0.19 *$ & 13.450 & 0.000 \\
\hline RMS-coma $(\mu \mathrm{m})$ & $0.38 \pm 0.22$ & $0.38 \pm 0.21$ & $0.34 \pm 0.21$ & 1.178 & 0.309 \\
\hline RMS-SA( $\mu \mathrm{m})$ & $0.42 \pm 0.16$ & $0.45 \pm 0.21$ & $0.44 \pm 0.20$ & 0.681 & 0.507 \\
\hline Strehl Ratio & $0.16 \pm 0.04$ & $0.16 \pm 0.04$ & $0.19 \pm 0.05^{*}$ & 12.938 & 0.000 \\
\hline
\end{tabular}

*: The value was significantly different from that in the other two groups.

SMILE: small incision lenticule extraction; WFO: Wavefront-optimized laser in situ keratomileusis; TCAT: Topography-guided custom laser in situ keratomileusis; OPD: optical path difference; RMSh: root mean square of higher order aberrations; AST: corneal astigmatism; SA: spherical aberration

Table 4 Comparison of corneal aberrations and visual quality among the three groups at 6 months postoperatively

\begin{tabular}{|c|c|c|c|c|c|}
\hline & SMILE(n=102) & $\mathrm{WFO}(\mathrm{n}=100)$ & $\operatorname{TCAT}(n=81)$ & $F$ & $\mathbf{P}$ \\
\hline $\mathrm{OPD}(\mu \mathrm{m})$ & $0.91 \pm 0.26$ & $0.93 \pm 0.29$ & $0.81 \pm 0.27 *$ & 5.078 & 0.007 \\
\hline $\operatorname{RMSh}(\mu \mathrm{m})$ & $0.70 \pm 0.20$ & $0.73 \pm 0.23$ & $0.71 \pm 0.27$ & 0.462 & 0.631 \\
\hline RMS-AST $(\mu \mathrm{m})$ & $0.53 \pm 0.30$ & $0.52 \pm 0.30$ & $0.34 \pm 0.19 *$ & 13.547 & 0.000 \\
\hline RMS-coma $(\mu \mathrm{m})$ & $0.40 \pm 0.20$ & $0.40 \pm 0.22$ & $0.38 \pm 0.22$ & 0.330 & 0.719 \\
\hline RMS-SA( $(\mu \mathrm{m})$ & $0.45 \pm 0.16$ & $0.47 \pm 0.19$ & $0.47 \pm 0.20$ & 0.414 & 0.662 \\
\hline Strehl Ratio & $0.16 \pm 0.04 *$ & $0.17 \pm 0.06 *$ & $0.19 \pm 0.05^{*}$ & 9.450 & 0.000 \\
\hline
\end{tabular}

*: The value was significantly different from that in the other two groups.

SMILE: small incision lenticule extraction; WFO: Wavefront-optimized laser in situ keratomileusis; TCAT:

Topography-guided custom laser in situ keratomileusis; OPD: optical path difference; RMSh: root mean square of higher order aberrations; AST: corneal astigmatism; SA: spherical aberration

\section{Figures}




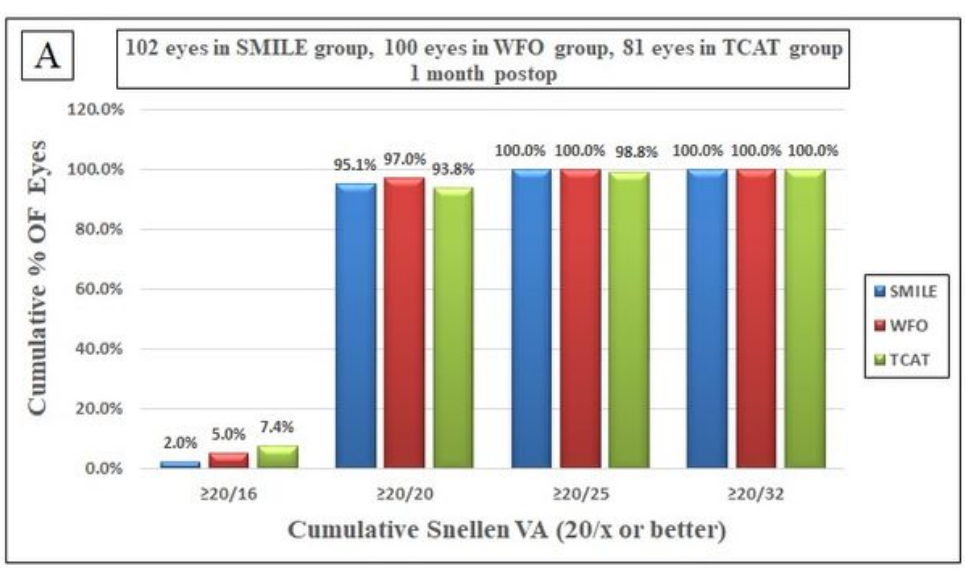

Uncorrected Distance Visual Acuity

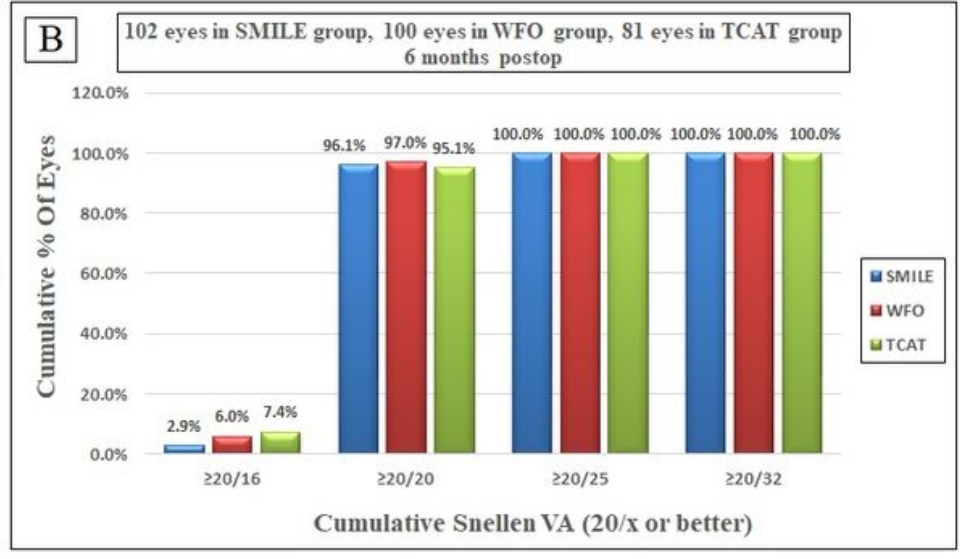

Uncorrected Distance Visual Acuity

\section{Figure 1}

Uncorrected distance visual acuity 1 month (A) and 6 months $\llbracket B$ Bafter surgery in the SMILE group, wavefront optimized (WFO) group and topography-guided custom ablation treatment (TCAT) group.

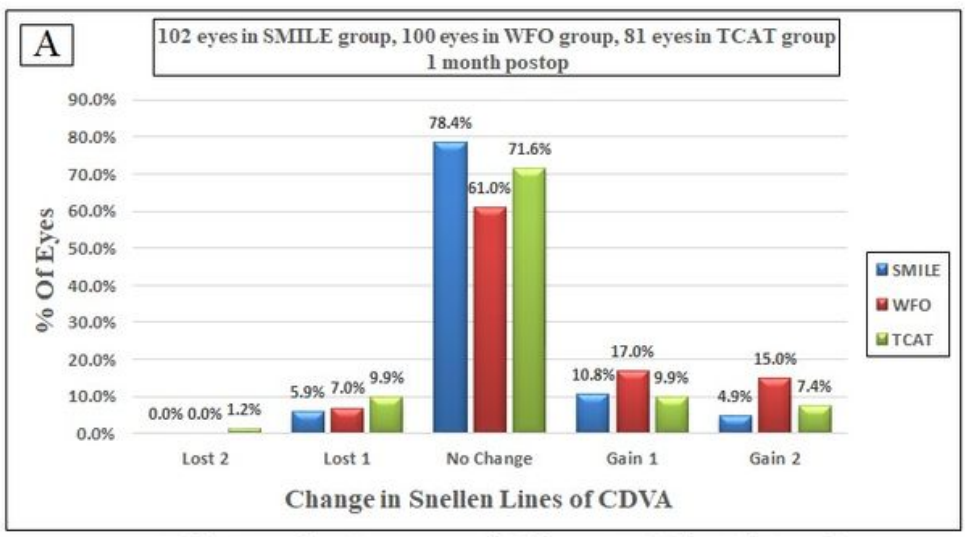

Change in Corrected Distance Visual Acuity

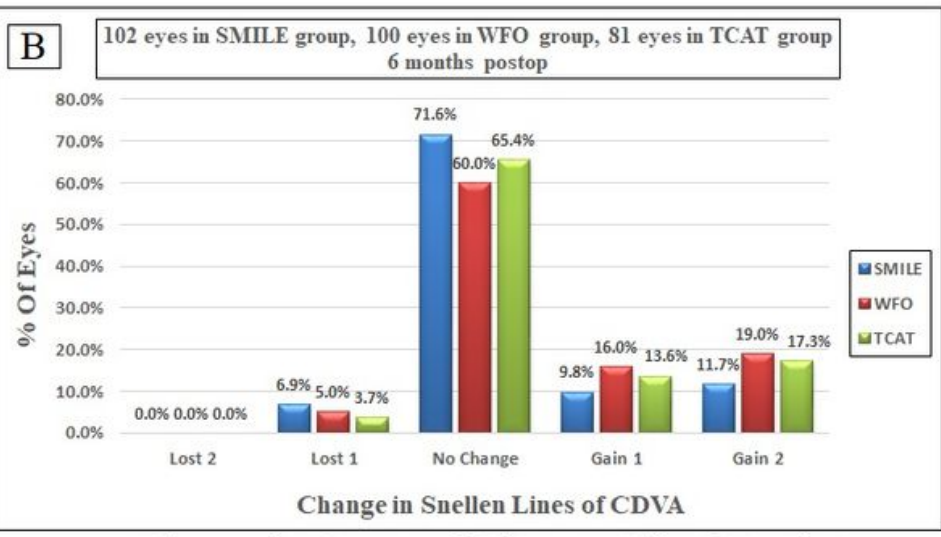

Change in Corrected Distance Visual Acuity

\section{Figure 2}

Change in Snellen lines of corrected distance visual acuity 1 month $(A)$ and 6 months $₫ \mathrm{~B} \bigotimes$ after surgery in the SMILE group, wavefront optimized (WFO) group and topography-guided custom ablation treatment (TCAT) group. 


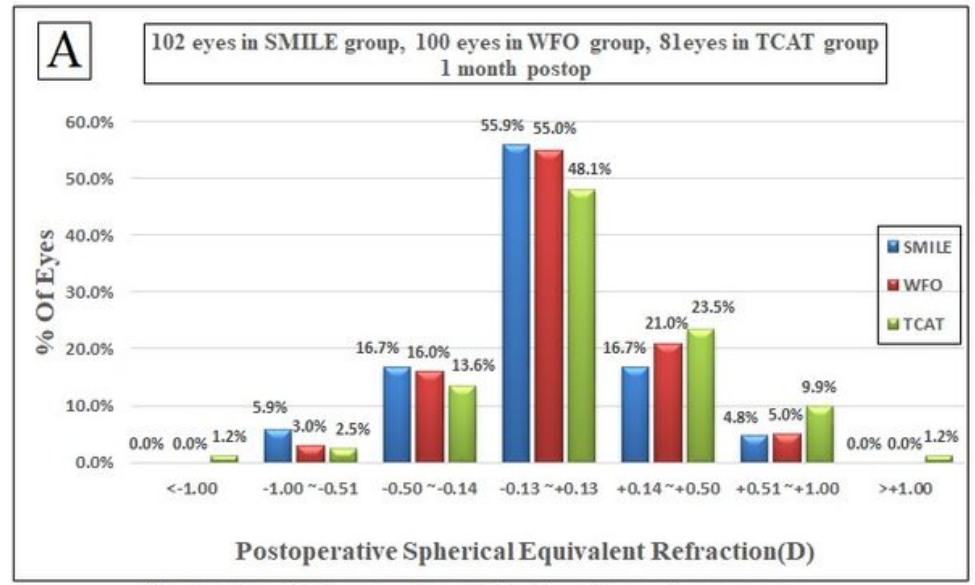

Spherical Equivalent Refractive Accuracy

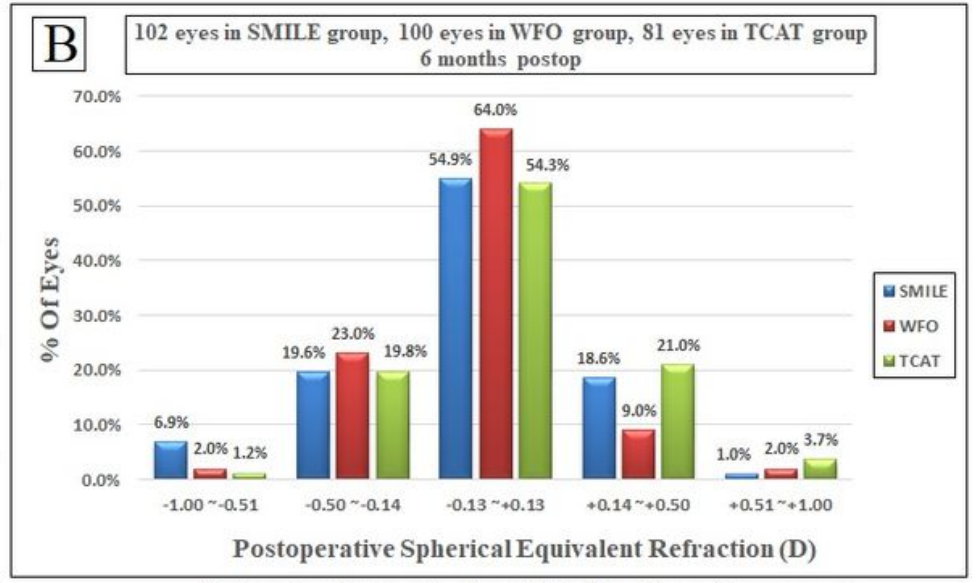

Spherical Equivalent Refractive Accuracy

\section{Figure 3}

Spherical equivalent refractive accuracy 1 month $(A)$ and 6 months $₫ B \bowtie$ after surgery in the SMILE group, wavefront optimized (WFO) group and topography-guided custom ablation treatment (TCAT) group.

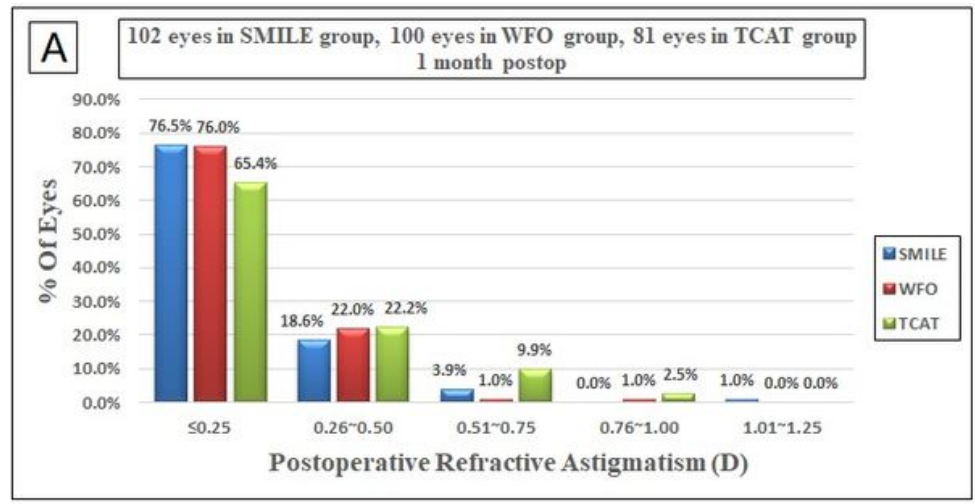

Refractive Astigmatism Accuracy

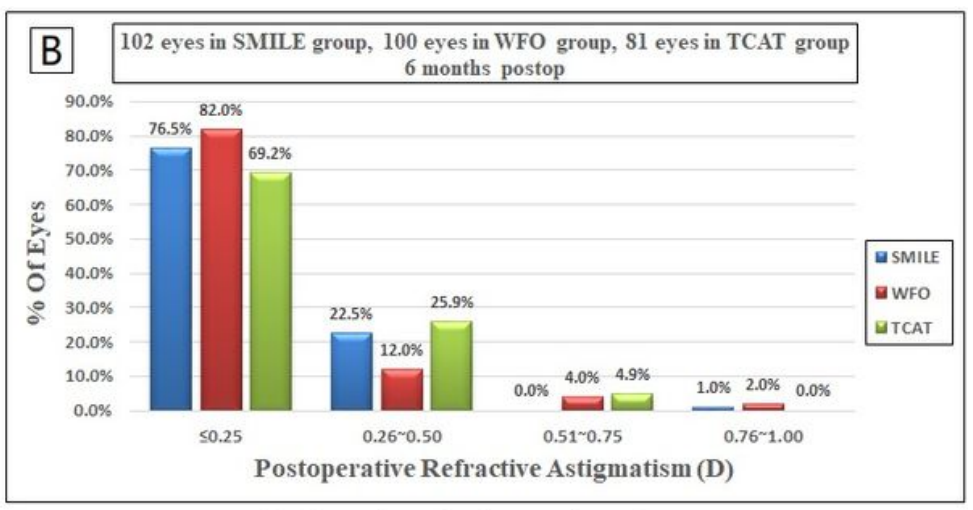

Refractive Astigmatism Accuracy

\section{Figure 4}

Refractive astigmatism accuracy 1 month (A) and 6 months $囚 \mathrm{~B} \bigotimes$ after surgery in the SMILE group, wavefront optimized (WFO) group and topography-guided custom ablation treatment (TCAT) group. 